\title{
Enhance Data Collection Process of a UAV-aided Low Power IoT Wireless Sensor Network
}

\author{
Pasan Samarakkody*, Sachith Guruge ${ }^{\dagger}$, Dulaj Samaradeera ${ }^{\ddagger}$, Eranda Jayatunga ${ }^{\S}$, Pawani Porambage ${ }^{\Uparrow}$ \\ ${ }^{*} \dagger £$ Department of Electrical and Information Engineering, University of Ruhuna, Galle, Sri Lanka \\ "Centre for Wireless Communications, University of Oulu, Finland \\ $\left\{{ }^{*}\right.$ pasanmalinda96, ${ }^{\dagger}$ sachithguruge, ${ }^{\ddagger}$ lakshan.dulaj94\}@ gmail.com, ${ }^{\S}$ eranda@eie.ruh.ac.lk, ${ }^{\Uparrow}$ pawanilp@ gmail.com
}

\begin{abstract}
Amalgamation of low power IoT devices in Wireless Sensor Networks (WSNs) has enhanced the usage of WSNs as it lessens the requirements of total power consumption. Most such IoT devices comparatively employ short-range radio access technologies such as Bluetooth Low Energy (BLE) to transfer sensor data. When a WSN is situated in a remote region such that neither regular human monitoring is plausible nor Internet coverage is readily available, it requires an alternative method to gather data from sensor nodes. Hence, in this research, an attempt was made to improve the data collection process and monitor the parametric performance of a low power IoT WSN aided by an Unmanned Aerial Vehicle (UAV). The impact of physical parameters related to different sensor node arrangements was investigated to effectively utilize the total flying time of the drone. Subsequently, adjustments were proposed for improvements by referring to previous literature. The Hamiltonian path defined in Graph Theory was applied to reduce the traverse distance of the drone further by tracing the sensor nodes within the grid area systematically. A prototype was implemented using commercially available devices to demonstrate the feasibility and the functionality of the proposed solution. Our results show that the proposed system increases the data collection performance by $23 \%$ compared to referenced architecture.

Index Terms-Internet of Things (IoT), Wireless Sensor Network (WSN), Unmanned Aerial Vehicle (UAV), Bluetooth Low Energy (BLE)
\end{abstract}

\section{INTRODUCTION}

The Internet of Things (IoT) is a modern advanced evergrowing network of physical objects featuring Internet connectivity, which offers communication between the objects and other Internet-enabled devices and systems. Undergoing a transition in recent years from an experimental technology, it promises things being able to connect to any place or anyone at any time, ideally using any network or service. Nowadays, low power IoT devices are gaining in popularity for wireless sensor networks (WSNs), due to power consumption being an important parameter in WSN, apart from others such as data rate, storage and sensor node arrangement. Therefore, more practical and efficient deployment of low power IoT devices in appropriate use cases are encouraged, which include industrial automation, agriculture, environment monitoring, smart grid infrastructure, smart home and smart city applications [1]. Most low power IoT devices in the industry can be used with short range radio access technologies, including Bluetooth Low Energy (BLE), IPv6 over Low-power Wireless Personal Area Networks (6LoWPAN), Near-Field Communication (NFC) and ZigBee. However, the mostly used low- power communication technology is BLE, operated at the short range communication in $2.4 \mathrm{GHz}$ bandwith, provides low cost and low power features [2].

Drones have recently become a topic of interest for their potential in wireless communication applications, due to low cost and high mobility attributes. From the historical military applications, they have emerged in new areas such as weather monitoring, disaster management and communication relaying [3]. However, there is limited research conducted in the area of collaboration of drone systems and low power IoT devices. In this setting, a recent research by Rajakaruna et al., in [4], introduces a system model enabling end-to-end connectivity for low-power IoT devices with UAVs. Extensive study in the considered fields reveals that further research is required on this front. The rest of the paper is arranged as follows; Section II evaluates related literature and Section III presents the system model. The prototype implementation and results are presented in Section IV and V respectively. Finally, Section VI presents the conclusions from the research.

\section{RELATED WORK}

In the IoT paradigm, short range radio access technologies such as BLE have become a key enabling technology. Bluetooth Low Energy was also branded as 'Bluetooth Smart' upon its introduction in 2010 as part of Bluetooth 4.0 Core Specification. Unlike classic Bluetooth, it was designed as a radio standard with the lowest possible power consumption, and specifically optimized for low cost, low bandwidth, and low complexity. Operating in the industrial, scientific and medical (ISM) band, BLE has a connection time of a few milliseconds with a high data rate of $1 \mathrm{Mb} / \mathrm{s}$ before going into hibernation mode until a connection is re-established [2]. Although BLE network has a maximum linkable device limitation, it is still a light-weighted and commonly supported mobile protocol, making it well-suited for this study.

A WSN consists of a collection of low-powered, small devices, known as sensor nodes, which are capable of sensing the physical environment, collecting and processing sensed data, and communicating with each other to accomplish certain common tasks. The nodes are typically equipped with a microcontroller, a low-power radio, power unit, and a number of sensors [5]. Laubhan et al. [6] proposed a real-time IoT-based four layer WSN with configurable nodes and multiple sensors 
included. The WSN comprised of four layers, including sensors (layer 1), nodes (layer 2), hubs (layer 3), and cloud server (layer 4), with the nodes implemented as nRF24L01+ battery powered transceiver and the cluster hubs as Raspberry $\mathrm{Pi}$ 2 boards. Current consumption and communication variation testing had been conducted on the WSN.

The survey paper by Zeng et al. [3], provides a comprehensive overview of opportunities and challenges associated with wireless communication systems employing UAVs. Typical use cases of UAVs were also discussed, including UAVaided delay-tolerant data collection from a large number of distributed wireless devices, such as in precision agriculture applications. Porambage et al. [7] have presented a solution to establish end-to-end secure connectivity between low-power IoT sensors and the cloud server by employing a mobilebased relay. For ambient assisted living application, they have simulated and implemented E2E secure connectivity for CC2650 SensorTag nodes with the cloud server through mobile phone relays. It was concluded that the protocol works with intermittent relaying to the cloud about three times per hour, depending on data generation rate. However, for the proposed project, the relaying interval from WSN should ideally be larger. Rajakaruna et al. [4] introduced a system model which enables end-to-end secure connectivity between low-power IoT devices and UAVs, helping to manage the monitoring of a heterogeneous WSN. They have considered IoT devices with diversified sensors communicating through BLE and drone performing as an edge server. Simulations have been run through MATLAB for linear, circular and square orientations of sensor node arrays, on double-hop collection, to analyze impact of BLE data rate, sensor data rate, drone's speed, distance between sensors. The results showed that the overall system performance is mainly defined by the distance between sensor nodes, and that circular and square array type sensor node arrangements outperform the linear array topology. Limited project implementation had been carried out using BLE Waspmotes, Raspberry Pi 3 and DJI Phantom 3 SE drone.

In the case of a drone monitoring a WSN by travelling overhead while communicating with each node, graph theory could be employed for analysis by representing the WSN as a graph. A graph is a set of vertices (nodes or points) connected to each other by edges (arcs). Traversing a path through the graph, where the first vertex and the last vertex are the same, is called a cycle. A cycle that covers each vertex in the graph just once is known as a Hamiltonian cycle [8].

\section{System Model AND Simulation}

\section{A. System Architecture}

We propose a communication system aided by a UAV to monitor and collect data from a sensor network composed of low power IoT devices. The UAV communicates with the sensor nodes using a short-range radio access technology, BLE, and uploads its collected data to the central cloud on behalf of sensor nodes. The UAV visits sensor nodes one at a time while hovering above to retrieve data, and then moves to the next node, as illustrated in Figure 1. The motive for the selection of short-range radio access technology is solely depends on the application of interest when there is intermittent wireless connectivity.

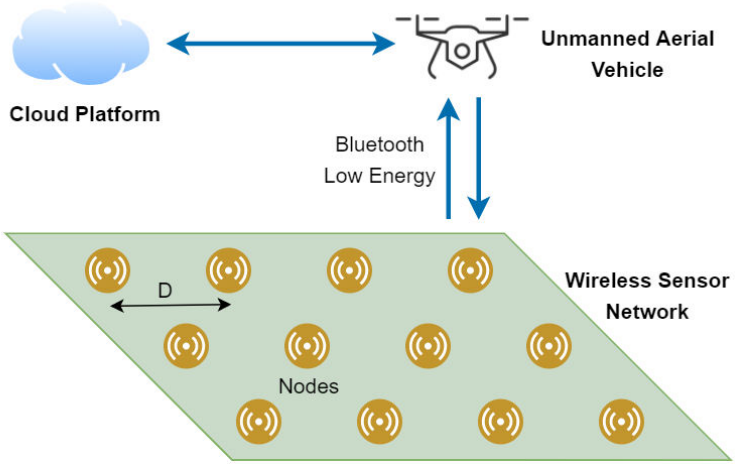

Fig. 1. System Architecture of Wireless Sensor Network and UAV

The sensor placement to cover the grid area of a particular application can be accomplished through various arrangements. The two most common arrangements for sensor node placement are linear and circular arrangements as shown in Figure 2. However, for a larger grid area, sensor nodes are uniformly arranged such that each sensor node has more than two closest neighbours located at equal distances. Examples for these arrangements include square arrangement [4], triangular-fill square arrangement and hexagonal arrangement. The square or triangular-fill square arrangements can be used to implement WSNs in areas having similar lengths and widths, whereas hexagonal arrangements can be used to cover areas more resembling to a circular region.

The distance that must be traversed by UAV, for each node arrangement, to cover and communicate all the sensor nodes above is compared in Table I. The parameters $n$ and $D$ denotes the number of sensor nodes and the distance between two sensor nodes respectively. Moreover, $\sqrt{n}$ is considered as an odd integer for square arrangements.

TABLE I

DISTANCE TRAVERSED BY DRONE IN DIFFERENT SENSOR NODE ARRANGEMENTS

\begin{tabular}{|l|c|}
\hline Sensor Arrangement & Total Distance Traversed $\left(D_{T}\right)$ \\
\hline Linear & $2 D(n-1)$ \\
\hline Circular & $D \times n$ \\
\hline Square & $D(n-1)+D\left(\frac{\sqrt{ } n-1}{\sqrt{ } 2}\right)$ \\
\hline Triangular- fill square & $D(n-1)+\frac{\sqrt{ } 7}{4} D(\sqrt{ } n-1)$ \\
\hline Hexagonal & $D(n-1)+\frac{D}{2}\left(\sqrt{\frac{(4 n-1)}{3}}-1\right)$ \\
\hline
\end{tabular}

1) Calculation of total flying time of drone - Referenced Architecture: The following expressions were used by Rajakaruna et al. [4] to define the total flying time $T_{\mathrm{F}}$ and the total waiting time $T_{\mathrm{W}}$ of the drone, where the drone communicates with a node only when hovering directly above. 


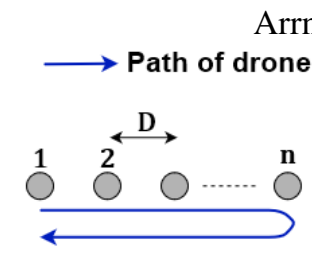

Linear arrangement

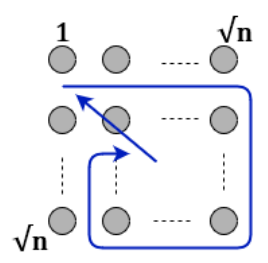

Square arrangement

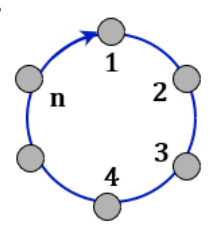

Circular arrangement

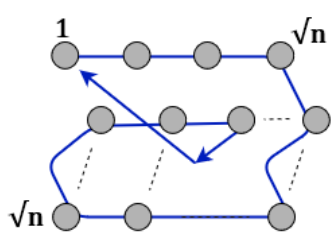

Triangular-fill Square arrangement

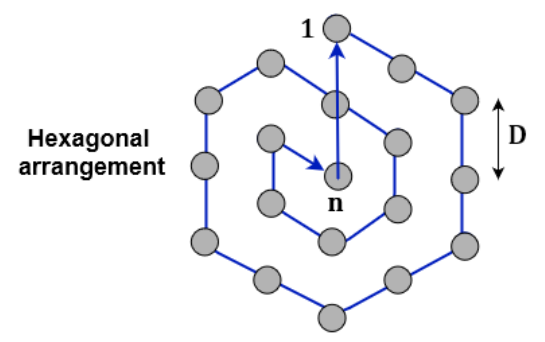

Fig. 2. Sensor Node Arrangements in WSNs

$$
\begin{gathered}
T_{F}=\left(n \times T_{W}\right)+\frac{D_{T}}{S} \\
T_{W}=\frac{T_{P}}{60}+\frac{T_{D L}}{60}+\frac{\left(R_{S}\left(T_{c}+T_{F}\right)\right)}{R_{B L E}}
\end{gathered}
$$

In these derivations, $D_{\mathrm{T}}$ is the total distance travelled by drone with a speed of $S$, and $T_{\mathrm{P}}$ is the processing time of the drone while the time required to download data from drone to node is $T_{\mathrm{DL}}$. The charging time of the drone is $T_{\mathrm{C}}$. Moreover, $R_{\mathrm{S}}$ and $R_{\mathrm{BLE}}$ denotes the sensor data generation rate, and the BLE data transfer rate between node and drone respectively. Then, the performance of the UAV-WSN system was evaluated based on the relationship between the number of covered sensor nodes $n$ and the total flying time $T_{\mathrm{F}}$, for linear and circular sensor arrangements.

2) Calculation of total flying time of drone - Proposed Architecture: The proposed system was built to lessen the observed shortcomings in [4], thus to improve the performance by selection of proper node arrangement. Therefore, we propose a novel method for the drone to communicate with a node before reaching the hovering point. This can be accomplished when the drone initially comes within the range of BLE communication is plausible, as shown in Figure 3.

As a result, the total data upload time $\left(T_{\mathrm{DU}}\right)$ from the ground node to the drone can be decomposed into two parts as upload duration before the hovering $\left(T_{\mathrm{DU} 1}\right)$ and upload duration during the hovering $\left(T_{\mathrm{DU} 2}\right)$ respectively. Therefore,

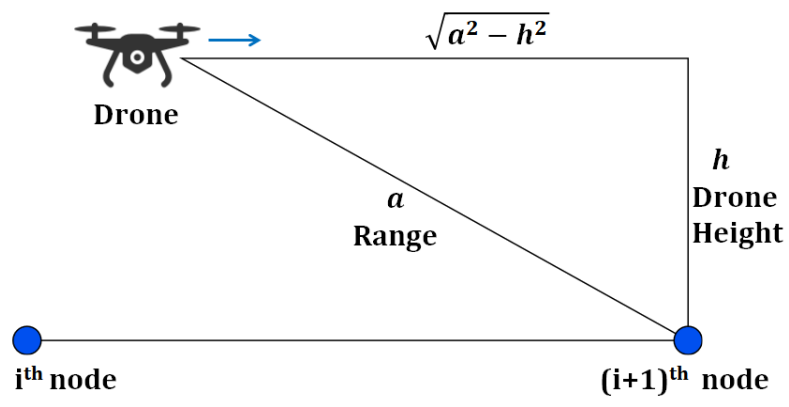

Fig. 3. Proposed data collection method with BLE

considering the geometry of the proposed model, the required upload time to aid the hovering process can derived as

$$
T_{D U 2}=T_{D U}-\frac{\sqrt{a^{2}-h^{2}}}{S}
$$

where $a$ is the maximum range between drone and node to initiate BLE communication and $h$ is the height of the drone from the ground. Apparently this reduces the waiting time of the drone thus expediting the process of retrieving data from sensor nodes. Consequently, the new waiting time of drone of the proposed system can be obtained by substituting $\left(T_{\mathrm{DU} 2}\right)$ in equation (4).

$$
T_{W, \text { new }}=\frac{T_{P}}{60}+\frac{T_{D L}}{60}+\left[\frac{R_{S}\left(T_{C}+T_{F}\right)}{R_{B L E}}-\frac{\sqrt{a^{2}-h^{2}}}{60 S}\right]
$$

\section{B. Proposed System Simulation}

Simulations were conducted through MATLAB simulator for the proposed architecture as well as the referenced architecture. The same simulation parameters were employed in both cases as tabulated in Table II.

TABLE II

SIMULATION PARAMETERS FOR PROPOSED SYSTEM

\begin{tabular}{|l|c|}
\hline Parameter & Simulation value \\
\hline Distance between nodes $(\mathrm{D})$ & $100 \mathrm{~m}$ \\
\hline Total flying time $\left(T_{F}\right)$ & $25 \mathrm{~min}[9]$ \\
\hline Charging time $\left(T_{C}\right)$ & $90 \mathrm{~min}[9]$ \\
\hline Speed of drone $(S)$ & $12 \mathrm{~m} / \mathrm{s}$ \\
\hline Drone processing time $\left(T_{P}\right)$ & $1 \mathrm{~s}$ \\
\hline Download time $\left(T_{D L}\right)$ & $10 \mathrm{~s}$ \\
\hline Sensor data generation rate $\left(R_{S}\right)$ & $20 \mathrm{~B} / \mathrm{min}[4]$ \\
\hline BLE data transfer rate $\left(R_{B L E}\right)$ & $10.5 \times 10^{4} \mathrm{~B} / \mathrm{min}[4]$ \\
\hline Drone height from ground $(h)$ & $10 \mathrm{~m}$ \\
\hline BLE communication range $(a)$ & $40 \mathrm{~m}$ \\
\hline
\end{tabular}

\section{Reducing the Traverse Distance of Drone using Hamilto- nian Paths}

In order to improve the data collection process further, an application of graph theory can be used for the sensor network traversal. For a square grid graph of $m \times m$, where $m$ is an even integer, it can be observed that all nodes can be traced by starting from the very first node in the first row, 
and then traversing along each row in alternate directions as shown in Figure 4. However, in this method, the nodes of the first column except the first row, must be avoided until it traces the nodes in the last row. Finally, the remaining nodes of the first column in other rows are traced. Such a traversal is known as a Hamiltonian path in graph theory [8]. A similar approach can be followed even for a hexagonal arrangement by traversing, first from the center node and then traversing outward along the hexagonal bands as shown in Figure 4. Hence, it can be shown that by using Hamiltonian paths, the total distance traversed in square, triangular-fill square arrangements (with an even $\sqrt{n}$ integer, where $\sqrt{n}=m$ ), or in hexagonal arrangement, is reduced to the same travel distance of a basic circular arrangement $(D \times n)$, thus gives the best node coverage number under a given flying time.

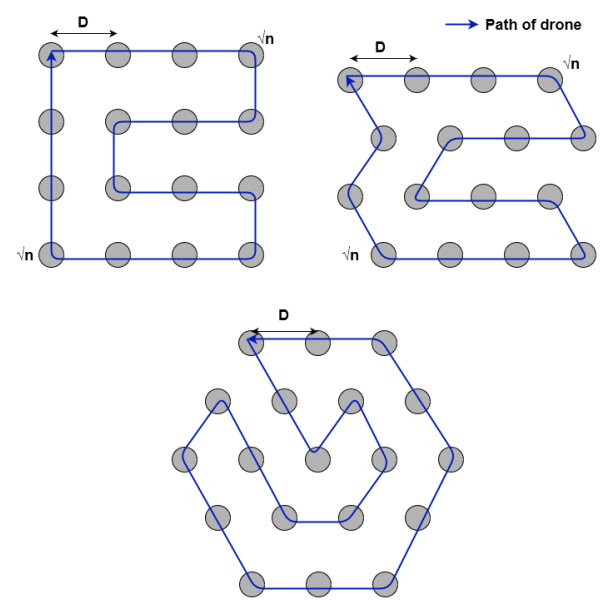

Fig. 4. Traversal of Sensor Node Arrangements using Hamiltonian Paths

\section{Modelling the BLE Link in Proposed System}

An analysis was conducted to determine a practically applicable BLE communication range for the system. In general, the signal attenuation in BLE is expressed with Received Signal Strength Indicator (RSSI) value. Therefore, the signal attenuation at a distance $d$ can be determined by employing the commonly used logarithmic path loss model [7].

$$
R S S I_{d}=R S S I_{d 0}+10 n_{0} \log \left[\frac{d}{d_{0}}\right]+X_{\sigma}
$$

where $\mathrm{RSSI}_{d}$ and $\mathrm{RSSI}_{d_{0}}$ are RSSI values in $\mathrm{dBm}$ at distance $d$ and reference distance $d_{0}$ respectively, expressed in meters. $X_{\sigma} \sim\left(0, \sigma^{2}\right)$ is the zero-mean Gaussian noise variable with variance $\sigma^{2}$. Normally, the path loss exponent $n_{0}$ depends on several environmental factors and presence of obstacles. Therefore, an open field test was conducted using CC2650 SensorTag modules and Raspberry Pi 3 development board to verify the applicability and validity of simulations. Note that we used the same values, as used in [10], i.e., $n_{0}=2.1$ and $\sigma^{2}=0.1$ for the BLE test.

\section{PRototype Implementation}

For the prototype implementation, we employed multiple CC2650 SensorTag modules, a Raspberry Pi 3 B development board and a Phantom 3 Standard drone [9] as the main apparatus. The implementation setup is shown in Figure 5.

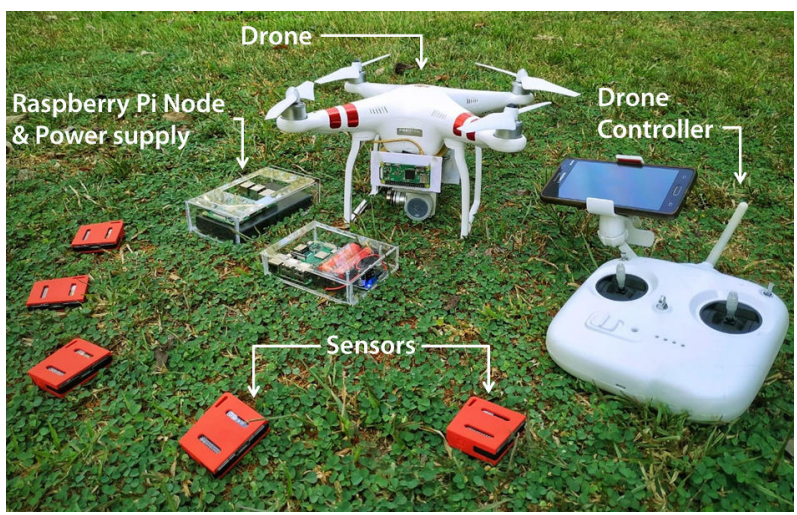

Fig. 5. Prototype Implementation of UAV-WSN System

The WSN was implemented using CC2650 SensorTag modules introduced by Texas Instruments, which support BLE, operate on coin cell battery and include multiple on-board sensors such as light, temperature, humidity, pressure and sound sensors. The sensor network was setup in an open field on faculty premises under linear and circular arrangements as shown in Figure 6, with the nodes being spaced $100 \mathrm{~m}$ and $50 \mathrm{~m}$ apart in each arrangement respectively.

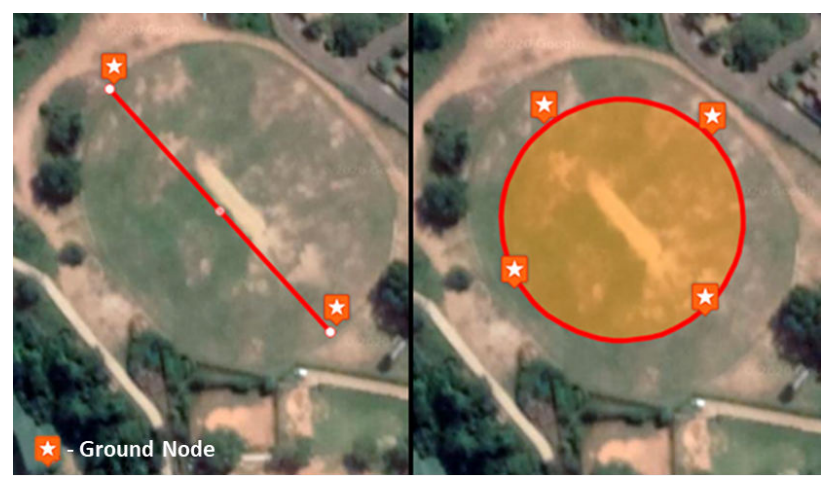

Fig. 6. Satellite View of Sensor Nodes in Linear and Circular Arrangements

Raspberry Pi 3 module with a $5.1 \mathrm{~V}$ power input from a portable power supply was used as the drone processor. It consisted of a Li-Ion rechargeable battery providing $7 \mathrm{~V}$ and a buck converter to step down the voltage to the appropriate value. The commercially available Phantom 3 Standard drone by DJI was chosen because of its payload capability upto 750 $\mathrm{g}$, without any significant strain on the propeller motors.

The SensorTag modules which functioned as sensor nodes were configured using Code Composer Studio 8.3. The BLE stack 2.2.3 was configured on CC2650 SensorTag with the parameters shown in Table III.

The Raspberry Pi 3 was configured using Bluez library for BLE communication. A Python script was run on the 
TABLE III

BLE Configured Settings For CC2650 SensorTag Modules

\begin{tabular}{|l|c|}
\hline Parameter & Configured value \\
\hline Advertising Interval & $100 \mathrm{~ms}$ \\
\hline Periodic event & $1000 \mathrm{~ms}$ \\
\hline Transmission Power & $0 \mathrm{dBm}$ \\
\hline Packet size & 18 bytes \\
\hline
\end{tabular}

Raspberry Pi to scan for advertising BLE sensor nodes in the WSN, whose MAC addresses were included in the MAC address whitelist provided to the drone. Once an appropriate sensor node was discovered, the broadcast RSSI value was confirmed to be in the reliable range for BLE communication before initiating the connection. The collected sensor data was then saved on a local MySQL database on the Raspberry Pi.

The drone was setup to traverse along the sensor network and to collect data from each node. Once the drone enters a region with Internet connectivity, it initiates a connection to the cloud to store the data. The cloud infrastructure needed for the prototype was implemented using the Google Firebase platform. The drone first authenticates itself with the cloud platform and then uploads the locally saved sensor data through HTTPS connection onto the real-time Firebase database.

\section{Results AnAlysis}

\section{A. Simulation Results}

The results of the MATLAB simulation of the proposed system was analyzed, including a comparison with the referenced architecture presented in [4]. Out of several evaluated parameters using the proposed UAV-WSN system, the distance between nodes was found to have the most pronounced impact on performance. Therefore, only the impact of distance between nodes $(D)$ on the performance of linear, circular, square, triangular-fill and hexagonal node arrangements are illustrated in Figure 8.

In line with the linear array of Figure 8, when the sensor nodes are apart by $50 \mathrm{~m}$, the drone can cover 69 sensor nodes, on average, in the referenced architecture for a total flying time of 25 minutes. In contrast, the equivalent value for the proposed system rises upto 85 nodes. Therefore, at minimum, $23 \%$ increase in data collection performance can be achieved for a linear array subject to aforesaid constraints. Other simulation results show that it is possible to obtain the same node coverage of circular array even with square, triangular-fill square and hexagonal arrangements, if the sensor nodes are traversed along Hamiltonian paths.

\section{B. Experimental Results}

Initially, experimental results were obtained from an open field test conducted using CC2650 SensorTag and Raspberry Pi 3 modules to study BLE range constraints. According to equation (5), the $\mathrm{RSSI}_{d_{0}}$ was measured to be $-59 \mathrm{dBm}$ for a transmitter of power level $0 \mathrm{dBm}$. RSSI readings of BLE communication were obtained at $5 \mathrm{~m}$ intervals, to verify

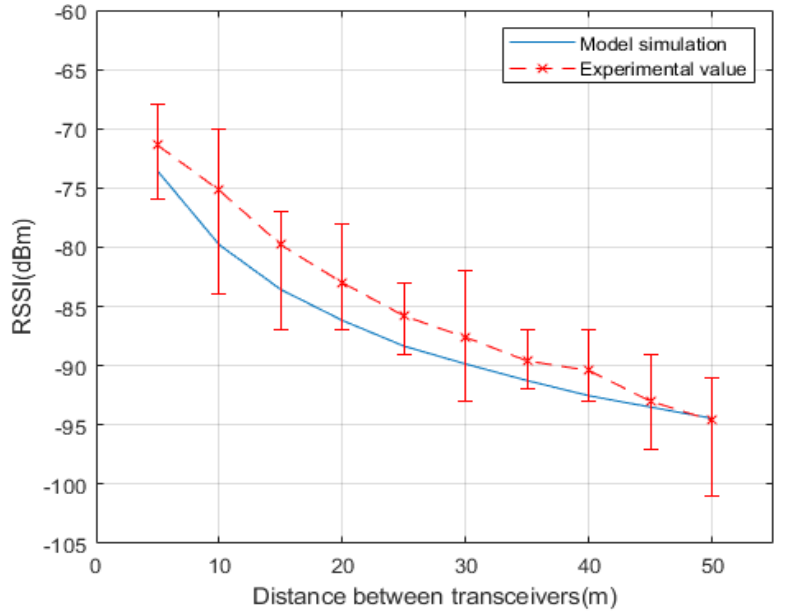

Fig. 7. Graph of BLE RSSI Test with Distance

the adequacy of simulations. Figure 7 illustrates that the experimental values for RSSI are consistent with the same obtained from the log normal model simulation. Considering a receiver threshold of $-97 \mathrm{dBm}$ [11] and a system margin of $3 \mathrm{dBm}$, the limit for most probable BLE communication range can be determined.

Using the prototype implementation, we were able to achieve the connectivity and data collection between the drone, cluster node and SensorTag modules. 10 repetitive tests were carried out for each node arrangement in order to determine the experimental parameters. On average, testing results showed that the drone takes 80 seconds for circular arrangement whereas 48 seconds for linear arrangement, to collect and upload the sensed data to the cloud. The plausible BLE range was tested using a total of 3 data sets of 10 transmission trials each. During the testing, a satisfactory successful transmission rate of $93.33 \%$ achieved with a BLE range of $40 \mathrm{~m}$, as shown in Table IV.

TABLE IV

RANGE RELIABILITY OF BLE LINK

\begin{tabular}{|l|c|c|c|}
\hline Height of Drone & $\mathbf{3 0} \mathbf{~ m}$ & $\mathbf{3 5} \mathbf{~ m}$ & $\mathbf{4 0} \mathbf{~ m}$ \\
\hline Success (out of 30) & 29 & 29 & 28 \\
\hline Success rate & $96.77 \%$ & $96.77 \%$ & $93.33 \%$ \\
\hline
\end{tabular}

\section{CONCLUSION}

The proliferation of low-power IoT devices in wireless sensor networks due to reduced power consumption, has widened the horizons of IoT. Throughout the paper, we have addressed the widespread accessibility and data collection process of a remote IoT based WSN with an UAV. The impact of different parameters related to performance of data collection and monitoring of the UAV-WSN system was evaluated and proposed improvements to previous literature. The simulations show that distance between sensor nodes is the dominant parameter in the data collection performance of the WSN. Introduction of the proposed system has led to an increase of data collection 
(A)

\section{Referenced}

Architecture

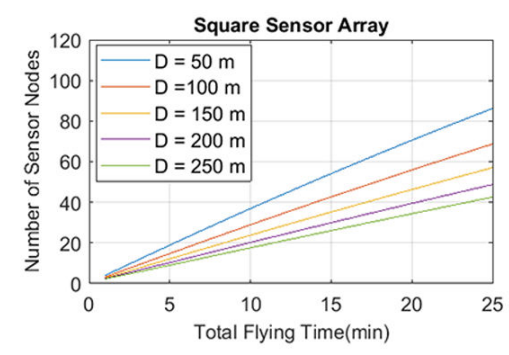

(B)

Proposed Architecture

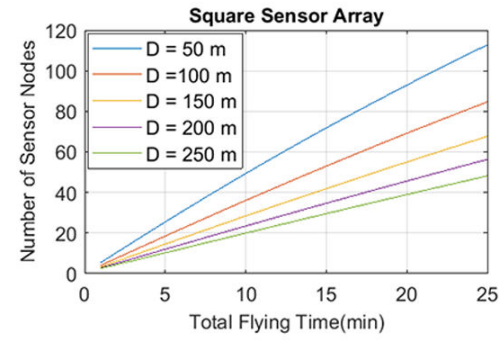

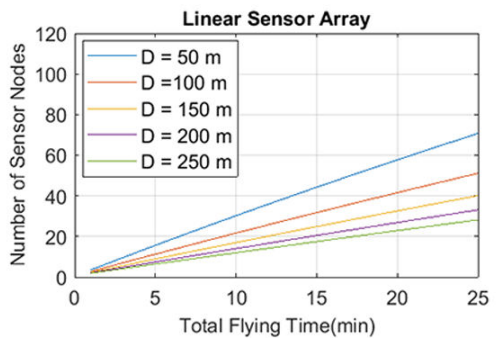
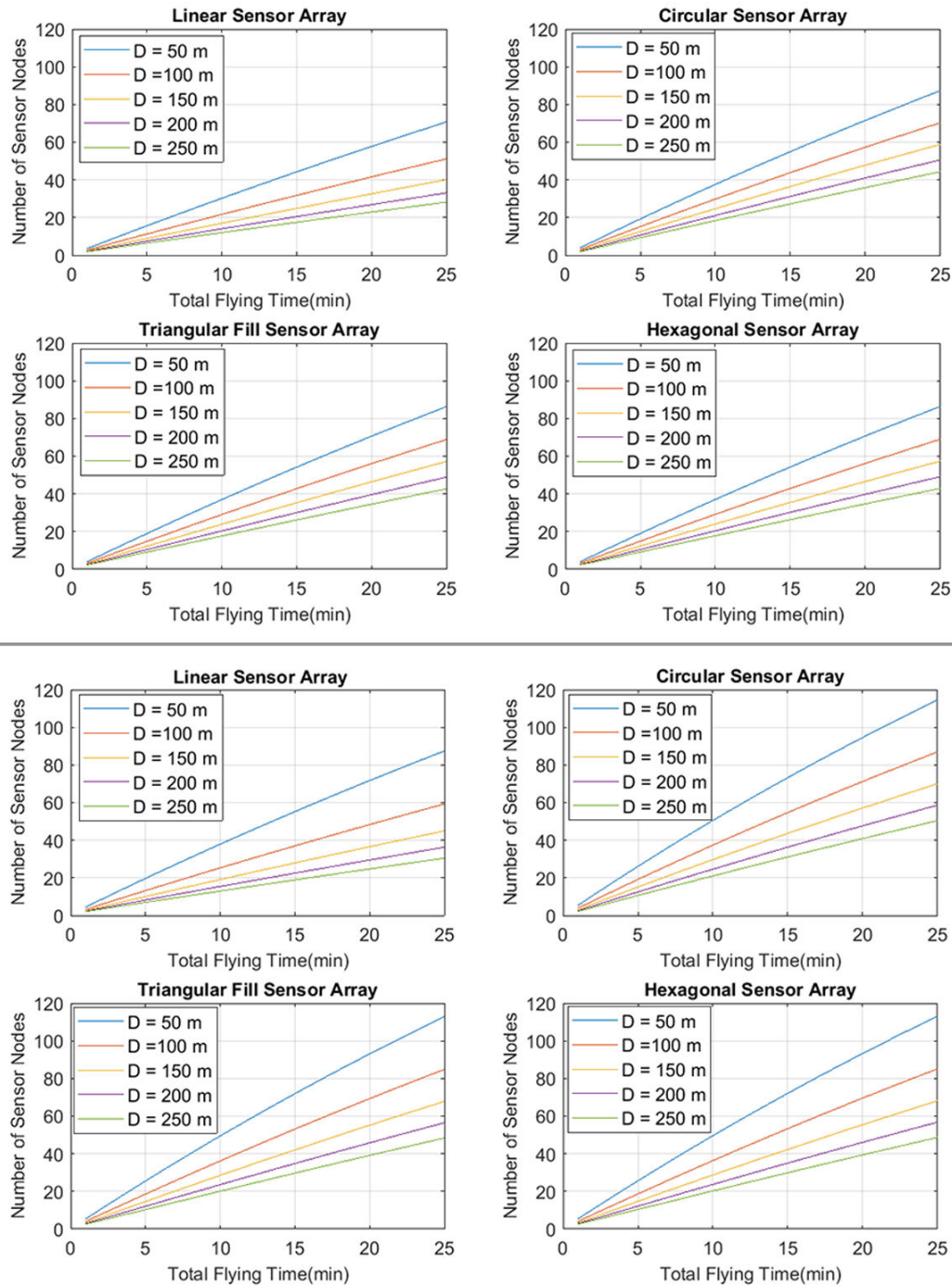

Fig. 8. Impact of Distance between Sensor Nodes in A) Referenced Architecture B)Proposed Architecture

performance by $23 \%$. Using Hamiltonian paths, it was shown that the traverse distance of drone in square, triangular-fill square and hexagonal arrangements can be further reduced upto the minimal distance akin to a circular arrangement. A prototype was implemented and tested for the proposed system using a Raspberry Pi module embedded to a Phantom 3 Standard drone and CC2650 SensorTag platforms using BLE communication. As future work, this system could be extended into a multi-UAV WSN monitoring system, utilizing a network of UAVs communicating with each other and ground nodes to monitor a particular sensing region.

\section{REFERENCES}

[1] C. Siu and K. Iniewski. IoT and low-power wireless: circuits, architectures, and techniques. CRC Press, 2018.

[2] K. Townsend. Getting Started with Bluetooth Low Energy. O'Reilly, 2014.

[3] Y. Zeng, R. Zhang, and T. J. Lim. Wireless communications with unmanned aerial vehicles: opportunities and challenges. IEEE Communications Magazine, 54(5):36-42, May 2016.
[4] A. Rajakaruna, A. Manzoor, P. Porambage, M. Liyanage, M. Ylianttila, and A. Gurtov. Enabling end-to-end secure connectivity for low-power iot devices with uavs. In 2019 IEEE Wireless Communications and Networking Conference Workshop (WCNCW), pages 1-6, 2019.

[5] H. Ammari. The Art of Wireless Sensor Networks: Volume 1: Fundamentals. Springer-Verlag Berlin Heidelberg, 2014.

[6] K. Laubhan, K. Talaat, S. Riehl, T. Morelli, A. Abdelgawad, and K. Yelamarthi. A four-layer wireless sensor network framework for iot applications. In 2016 IEEE 59th International Midwest Symposium on Circuits and Systems (MWSCAS), pages 1-4, 2016.

[7] P. Porambage, A. Manzoor, M. Liyanage, A. Gurtov, and M. Ylianttila Managing mobile relays for secure e2e connectivity of low-power iot devices. In 2019 16th IEEE Annual Consumer Communications Networking Conference (CCNC), pages 1-7, 2019.

[8] William F Mitchell. Hamiltonian paths through two-and threedimensional grids. Journal of Research of the National Institute of Standards and Technology, 110(2):127, 2005.

[9] Phantom 3 Standard. https://www.dji.com/phantom-3-standard/info. [Online; Accessed 02-January-2020].

[10] N. El Agroudy, N. Joram, and F. Ellinger. Low power rssi outdoor localization system. In 2016 12th Conference on Ph.D. Research in Microelectronics and Electronics (PRIME), pages 1-4, June 2016.

[11] CC2650. http://www.ti.com/product/CC2650. [Online; Accessed 03January-2020]. 